\title{
Integrated Supply of Hydrogen as Transporter of Future Green Energy
}

\author{
Gyan Ranjan Biswal $^{1} \quad$ R. P. Maheshwari ${ }^{2} \quad$ M. L. Dewal ${ }^{3}$ \\ Department of Electrical Engineering ${ }^{1,2,3}$ \\ Shiv Nadar University, Noida ${ }^{1}$ and Indian Institute of Technology, Roorkee $e^{2,3}$
}

\begin{abstract}
A comprehensive hydrogen processing unit $(H P U)$ is presented for uninterrupted supplying of it at desired pressure rate. The pressurized Hydrogen is used for the cooling of large size generators in integrated gasification combined cycle power plants. It can also be seen as one of the future sources of fuel for automobile applications. Thereby, it reduces the chances of green house gases emission. Equally, Hydrogen can be utilized as a future source of energy for the automobiles. A novel HPU clubbed with highly reliable and efficient process automation system is proposed. The work includes a comparison between the proposed and the existing systems in terms of system reliability. The algorithm for system reliability is developed on MATLAB platform. The effectiveness of real-time featured proposed HPU is validated by computer simulation. The entire process automation system is designed and simulated on FTView32 Works. It meets the specifications guided by IEEE C37.1-2007, IEC 61131-3 and IEEE 14132010.
\end{abstract}

\section{Introduction}

In recent scenario, Reliability is a main impact index for performance quality of a plant / process. The designer's prime objective is to carry out the process with utmost overall efficiency of the system having maximum availability at minimum cost. The system reliability of control and instrumentation (C\&I) for HPU is of prime importance at the plant level primarily due to the inadequacies of component reliability. The system presented has enhanced system reliability of the process that reduces the chances of system failure. It requires less time for maintenance and providing higher availability. Further, reduction in maintenance duration of the process allows it to be kept in active mode for longer durations. In turn, the efficiency of overall system gets improved. Improved efficiency has its direct impact on reduction in emission of green house gases [1], [2], [3].

Now-a-days, the operations of fossil-fuel power plants require sophisticated automation system called supervisory control and data acquisition (SCADA) systems as they provide highly productive, reliable, and safe power generation. The SCADA systems have the capability to handle highly dynamic and composite structure like load distribution centers. Kawai et al. discussed about advanced methods and technologies involved in automated powergenerating plants [4]. Improvement in system reliability of plant processes is one of the areas of concern [5], [6].

The importance of maintenance function has increased because of its role in keeping and improving system availability and safety, as well as product / process quality. To support this role, the development of communication and information technologies has allowed the emergence of the concept of optimal maintenance [7]. Large size generating sections of a power plant are one of the critical components. For such components, SCADA operated highly reliable process C\&I system is necessary. Bannister et al. observed that efficiency of power plants can be enhanced by using generating energy from the combustion of hydrogen and oxygen [8]. Hydrogen is an excellent cooling agent for the cooling of large generators because of low density, high specific heat, and high thermal conductivity properties [9]. It is also known as a green gas [10]. It is a usual practice to store hydrogen in liquid form. Liquid hydrogen takes up less storage volume than gas but requires cryogenic vessels. It is expected that future $\mathrm{LH}_{2}$ storage systems will have shaped geometries made of lightweight materials [11]. In present paper, Weibull distribution function has been considered to evaluate the chances of system failure in such cases (like hydrogen cooling systems). Fault tree analysis (FTA) is a logical and structured process in industrial applications that can help to identify potential causes of system failure before the failures actually occur [12], [13]. In view of the need for greater system reliability and customer's satisfaction, the emphasis has been placed on cost reduction.

A digitally operated hydrogen cooling system was proposed by Hargrove et al. [14]. It is composed of an indirect heat exchanger for bringing cooling water into heat exchanger with hydrogen leaving the generator. In the follow up, Blatter et al. has also proposed a three stage cooling circuit for the cooling of generators [15]. Brosnihan et al. has furthered the work by proposing a modular system of air, hydrogen, and carbon dioxide through a gas 
manifold for monitoring of hydrogen cooled generators, where various modules employed are gas dryer, gas purity, overheat, and gas/generator monitoring modules [16]. The cost reduction issues are implicitly focused on reducing operation and maintenance expenses, and minimizing investment in new plant set up. Here, FTA plays a key role to analyze system design and reliability of a dedicated process model. Biswal et al. proposed a process model of hydrogen which is reliable for the units of capacity up to 120-300 MW of a generating station [2], [3]. Such complex systems require redundant schemes which ensure reliable and uninterrupted operations.

Recent optimization techniques are implemented to develop the hydrogen supply chain for the transport sector. These techniques are used to determine the optimum infrastructure and operational costs [17]. While considering hydrogen as an alternative vehicle fuel, is gaining increasing attention internationally, one significant aspect of its introduction has been given less attention than others; the public acceptance of such a new technology and fuel. The importance of understanding public acceptance for the successful introduction of new products and technologies has been demonstrated by Schulte et al. [18]. In the follow up, Xiao et al. developed an optimization algorithm for the hydrogen production schedule and the strategy of power trading to optimize the production costs of a station [19].

\section{Processing of Hydrogen}

Weibull's distribution described in equations (1)and-(2) is considered for all the systems; proposed and the existing, for resolving the design issues and evaluating the system reliability and bring out comparison between the proposed and the existing methods. For carrying out comparison at common platform, all the existing models and the one proposed are simplified with hot redundancy concept based on (3). Typical hazard rate $h(t)$ as given by equation (1) below is considered for the evaluation of system reliability based on philosophy of $k-o u t-o f-n: G$ with $k=1$ (hot unit) and $n=2$ (total number of units). Equations (1)-and-(2), represent relationships between reliability functions $\mathfrak{R}(t)$ and the hazard rate $h(t) . \mathfrak{R}(t)$ and $\mathfrak{R}_{s}(t)$ are the component and system reliability respectively, of the process model with hot redundant unit(s), in days. In the present paper, $\mathfrak{R}_{s}(t)$ is the overall system reliability of the processing model of hydrogen. ' $\beta$ ', is the slope parameter and ' $\eta$ ' is the scale parameter of the two-parameter Weibull distribution function.

$$
\left.\mathfrak{R}(t)=e^{-(t / \eta)^{\beta}} \text { and } h(t)=(\beta / \eta)^{*}(t / \eta)^{\beta-1}\right\}
$$

$\mathfrak{R}_{s}(t)=\sum_{i=0}^{k}\left\{\int_{0}^{t} h(t) d t\right\}^{i} e^{-\int_{0}^{t} h(t) d t}(i !)^{-1}=\sum_{i=0}^{k=1} \frac{(h t)^{i} e^{-h t}}{i !}(2)$

Here onwards, R5, R2, R3, and R4 are the corresponding system reliabilities of the systems proposed by the authors, Hargrove et al. [14], Blatter et al. [15], and Brosnihan et al. [16]. The major components of the processing units such as, heat exchangers, cold-converters, and reservoirs are considered to draw the reliability diagram for keeping the mathematical evaluations simple using Network Reduction Technique. Reliability is always an implicit function of time, however, values obtained of system reliability are the collection of samples at different instants of time, and thus, these values are discrete in nature. Heat exchangers are represented by $\left(R_{H E 1 A}\right.$ to $\left.R_{H E 4 B}\right)$, reservoir $\left(R_{T A}\right)$, processing tanks ( $R_{T B}$ and $\left.R_{T C}\right)$.

$$
\left.\begin{array}{l}
\mathfrak{R}_{s}(t)=1-(1-R(t))^{n}, \because \mathfrak{R}_{s}(t)_{\text {series }}=R_{p} * R_{q} \\
\text { and } \mathfrak{R}_{s}(t)_{h o t / \|}=R_{p} \| R_{q} \\
\therefore \mathfrak{R}_{s}(t)_{\|}=1-\left\{\left(1-R_{P}\right) *\left(1-R_{Q}\right)\right\}
\end{array}\right\}
$$

\section{Serial system}

It is the simplest system in terms of system reliability. The reliability model of the system is expressed in Eq. (4).

$\mathfrak{R}_{1}(t)=R_{T A} * R_{T B} * R_{H E 1} * R_{H E 2}=R 1$

\section{Hargrove et al. system}

Hargrove et al. introduced a system relating to the $\mathrm{H}_{2}$ cooled generators in an electrical power station [14]. The reliability model of the system is provided in (5).

$$
\left.\begin{array}{l}
\Re_{2}(t)=R_{X} * R_{T B} * R_{H E 1} * R_{H E 2}=R 2 \\
\because R_{X}=R_{T A} \| R_{S I V L}
\end{array}\right\}
$$

\section{Blatter et al. system}

Blatter et al. introduced a three stage hydrogen cooling system with a typical matrix of storage tanks for providing redundancy which acts as a reservoir to feed hydrogen [15]. The reliability model is expressed by:

$$
\left.\begin{array}{l}
\Re_{3}(t)=R_{T A} * R_{T B} * R_{\beta} * R_{\gamma}=R 3 \\
\because R_{\beta}=R_{\gamma}=R_{H E x k} \| R_{H E y k}, 1 \leq x, y \leq 2 \text { and } 0 \leq k \leq 1
\end{array}\right\}(6)
$$

\section{Brosnihan et al. system}

Brosnihan et al. proposed a modular system for the monitoring of hydrogen-cooled generator [16]. Reliability model of the system is provided in (7). 


$$
\Re_{4}(t)=R_{T A} * R_{T B} * R_{H E 1}=R 4
$$

\section{Proposed processing system of hydrogen}

The proposed method is designed and supported with hot redundant module for uninterrupted supply of $\mathrm{H}_{2}$. The reliability of the system improves with increasing number of redundant components. Thus, a careful evaluation is done on determining redundancy for designing process models. Fig. 1 depicts the processing unit presented in the paper. The final and intermediate steps are given in equations (8) and (9).

$\left.\begin{array}{l}R_{X^{\prime}}=1-\left\{\left(1-R_{T A}\right) *\left(1-R_{S I V L}\right)\right\}, \therefore R_{X^{\prime}}=R_{T A} \| R_{S I V L} \\ R_{Y}=R_{H E 1 A} \| R_{H E 1 B}, R_{Y}=R_{Z}\end{array}\right\}$

$$
\left.\begin{array}{rl}
\because & R_{S}=R_{Y} * R_{T B}, R_{T}=R_{Z} * R_{T C}, \therefore R_{\alpha}=R_{S} \| R_{T} \\
& R_{\beta}=R_{H E 3 A} \| R_{H E 3 B}=R_{\gamma} \\
\therefore & R_{\text {proposed }}(t)=R_{X} * R_{\alpha} * R_{\beta} * R_{\gamma}=\Re_{5}(t)=R 5
\end{array}\right\}
$$

In order to provide a required level of system reliability, overall equipment effectiveness (OEE) are included. Usually engineers try to achieve this level with minimal cost. The problem of total investment cost minimization, subject to reliability constraints, is well known as the redundancy optimization problem. The redundancy optimization problem for a multi-state system, which may consist of elements with different performance rates and reliability, is addressed as a problem of System Reliability Optimization (SRO). It is backed by bidirectional / two stage analyses approach, namely, system reliability evaluation and fault tree analysis in the scheme presented in this paper.

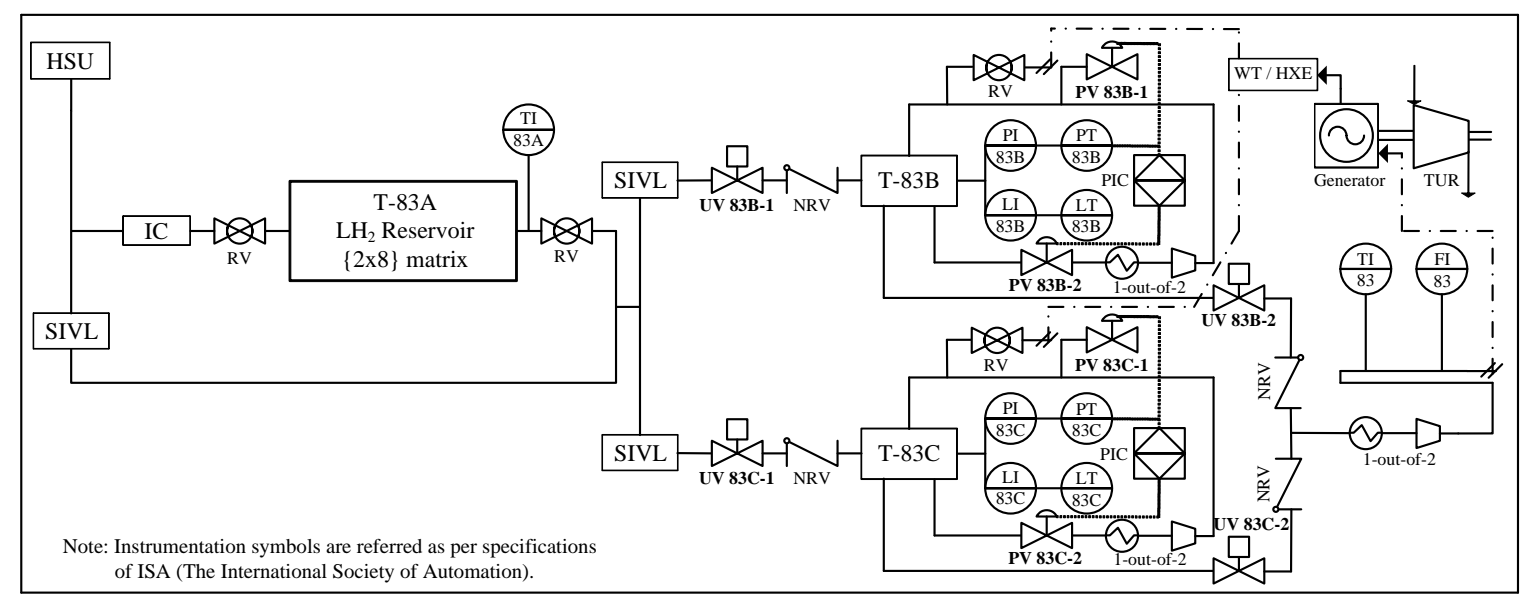

Figure 1. The schematic diagram of the proposed processing unit.

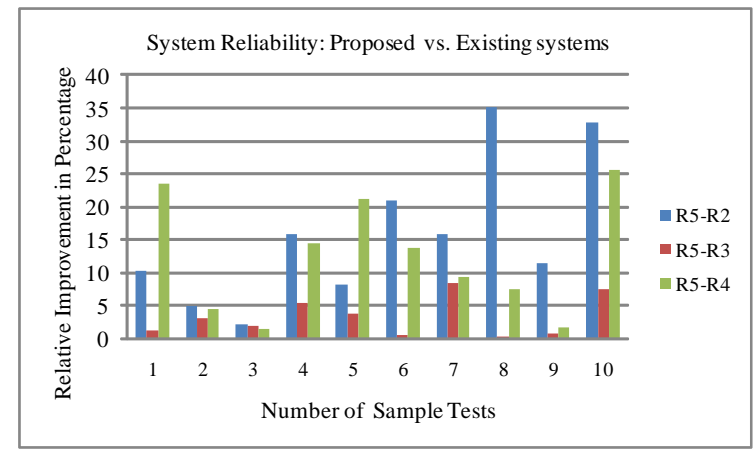

(a)

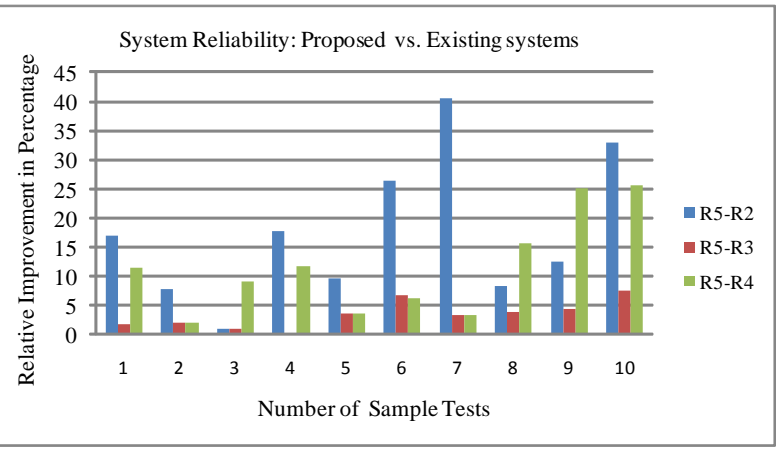

(b)

Figure 2. Histogram showing improvement in system reliability. 
TABLE I

Percentage improvement in System Performance

\begin{tabular}{|c|c|c|c|c|c|c|c|c|c|c|c|c|}
\hline \multirow{2}{*}{$\begin{array}{l}\text { (A) } \\
\text { No. of } \\
\text { tests } \\
\text { sample } \\
\text { s }\end{array}$} & \multicolumn{3}{|c|}{ (B) $\mathrm{F}_{\mathrm{S}}(\mathrm{t})$ of Test I (\%) } & \multicolumn{3}{|c|}{ (C) $\mathrm{F}_{\mathrm{S}}(\mathrm{t})$ of Test II $(\%)$} & \multicolumn{3}{|c|}{ (D) $\mathrm{R}_{\mathrm{S}}(\mathrm{t})$ of Test I (\%) } & \multicolumn{3}{|c|}{ (E) $\mathrm{R}_{\mathrm{S}}(\mathrm{t})$ of Test II (\%) } \\
\hline & F5 - F2 & F5 - F3 & F5 - F4 & F5 - F2 & F5 - F3 & F5 - F4 & R5 - R2 & R5 - R3 & R5 - R4 & R5 - R2 & R5 - R3 & R5 -R4 \\
\hline 1 & $\begin{array}{l}89.8 \\
\end{array}$ & 98.8 & 76.5 & 83.0 & 98.2 & 88.4 & 10.1 & 1.2 & 23.4 & 16.9 & 1.73 & 11.5 \\
\hline 2 & 95.1 & 97.0 & 95.5 & 92.1 & 97.8 & 98.0 & 4.86 & 2.96 & 4.5 & 7.89 & 2.11 & 1.92 \\
\hline 3 & 97.9 & 98.0 & 98.5 & 98.9 & 98.9 & 90.9 & 2.1 & 1.95 & 1.45 & 1.01 & 1.06 & 9.09 \\
\hline 4 & 84.1 & 94.5 & 85.5 & 82.2 & 99.6 & 88.2 & 15.8 & 5.44 & 14.46 & 17.8 & 0.34 & 11.7 \\
\hline 5 & 91.7 & 96.3 & 78.7 & 90.2 & 96.4 & 96.3 & 8.24 & 3.7 & 21.2 & 9.76 & 3.6 & 3.7 \\
\hline 6 & 79.1 & 99.3 & 86.3 & 73.5 & 93.3 & 93.6 & 20.8 & 0.61 & 13.6 & 26.4 & 6.69 & 6.37 \\
\hline 7 & 84.1 & 91.5 & 90.7 & 59.4 & 96.7 & 96.5 & 15.8 & 8.49 & 9.24 & 40.5 & 3.27 & 3.48 \\
\hline 8 & 64.8 & 99.8 & 92.5 & 91.7 & 96.0 & 84.2 & 35.1 & 0.17 & 7.46 & 8.26 & 3.98 & 15.7 \\
\hline 9 & 88.4 & 99.2 & 98.3 & 87.3 & 95.5 & 74.8 & 11.5 & 0.72 & 1.63 & 12.6 & 4.44 & 25.1 \\
\hline 10 & 67.1 & 92.4 & 74.3 & 67.1 & 92.4 & 74.3 & 32.8 & 7.51 & 25.6 & 32.8 & 7.51 & 25.6 \\
\hline
\end{tabular}

\section{System Reliability and System failure Analysis}

It is well known that 'system failure' is nothing but it is 'one minus system reliability', thus $F_{s}(t)=1-\mathfrak{R}_{s}(t)$ as depicted in Fig. 2; for example, $F 5=1-R 5$ and so on. It includes system generated values (samples 1-9) and threshold value $\left(10^{\text {th }}\right.$ sample) as shown in Table I and Fig. 2.

Table I illustrates percentage improvement in system performance of $\mathrm{R} 5$ in terms of $F_{s}(t)$ system failures, Col. B-and-C and $\mathrm{R}_{\mathrm{s}}(\mathrm{t})$ system reliability, Col. D-and-E vis-à-vis systems R2, R3, and R4. Col. A represents the number of samples collected at ten different instants of time. $10^{\text {th }}$ Test of $R_{S}(t)$ gives the difference between the system reliability of R5 and the system reliability of R2, R3, and R4 at the upper limit of threshold value. $R_{S}(t)$ at this threshold value is unique. Whereas, Test $1-9$ all are randomly generated values within the range of threshold values. From Table I; (a) Col. 4 represents $R_{s}(t)$ of Test I, and (b) Col. 5 represents $\mathrm{R}_{\mathrm{s}}(\mathrm{t})$ of Test II Similarly, Fig. 2 shows improvement in system reliability of R5 over R2, R3, and R4. (a)-and-(b) represent two different test sets Test I and Test II. To solve component failure issue, there are two approaches: improving mean time between failures (MTBF) and providing redundancy.

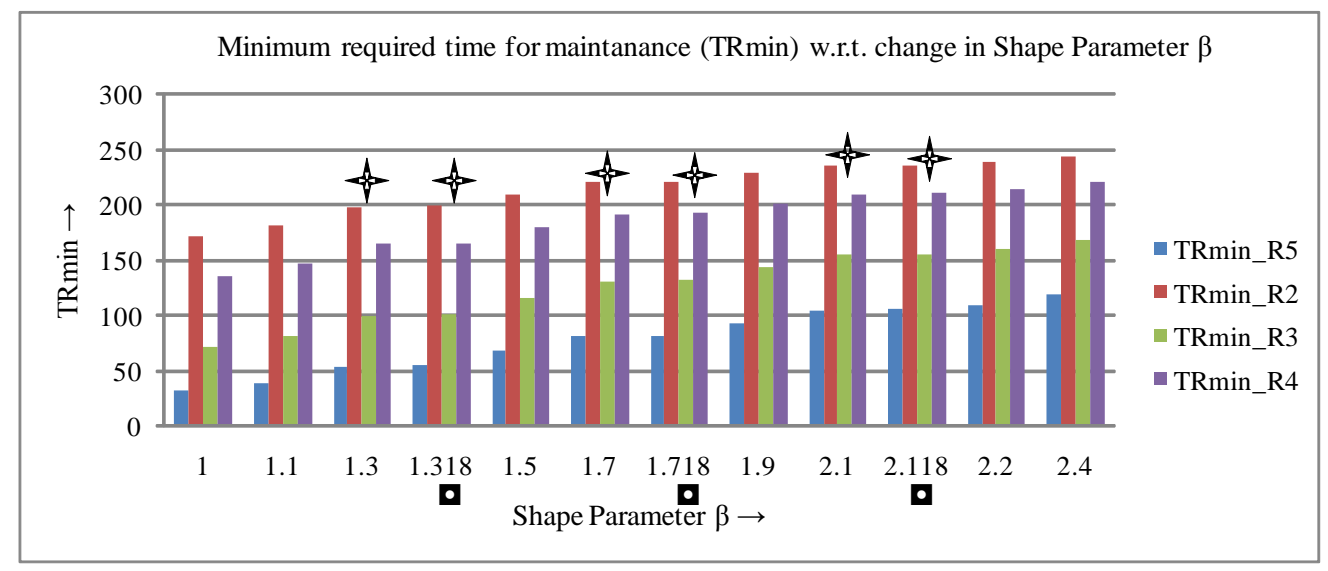

Figure 3. Histogram showing comparison between R5, R2, R3, and R4 models in terms of small variation in $\beta$ on the $T=T_{R \min }$ (in days) of these models. 
MTBF cannot change once a device is manufactured. Thus, in the proposed method, emphasis is given on hot redundancy, and considered the fault tolerance method for the analysis of system failures. In the present paper, Weibull's minimum reliable life $\left(T_{R}\right)$ which defines minimum reliable time required for maintenance is considered for evaluating the chances of system failure. The parameter is governed by (10).

$$
\left.\begin{array}{l}
T_{R}=\eta^{*}\left\{-\ln \left[R\left(T_{R}\right)\right]\right\}^{1 / \beta}, \text { if } R\left(T_{R}\right)=0.5 ; T_{R}=T^{\vee} \\
h(t)=1-e^{-(t / M T T R)}, \because T=-M T T R^{*}\{\ln [1-h(t)]\}
\end{array}\right\}
$$

The characteristic of $\mathrm{R} 5$ versus the existing systems in terms of TRmin is given in Fig. 3. The results of Test 5 as depicted in Fig. 3 indicate that the R5 needs minimum time for the maintenance than needed by the existing systems.

In general, fault avoidance methods prove to be less expensive than fault tolerance methods to evaluate system reliability. The fault avoidance methods and fault tolerance methods are used to evaluate the modal life of components and redundancy based improvement in system design, respectively. In case of fault avoidance methods, the cost increases exponentially with respect to linear improvement in reliability performance of the system. Also, it is observed that the behavior of components used as a part of the system can be different than the characteristics of components used as an individual. Thus, in industrial applications, one of the fault tolerance based methods is preferred to evaluate the system reliability of the present and the already existing systems.

There are three kinds of redundancy schemes, which can be used at system level integration. These are: standby redundancy; warm redundancy and hot redundancy.

- Standby (cold) redundancy: is for those processes where response time is of minimal concern, and may require operator intervention.

- Warm redundancy: is used where time is somewhat critical but a momentous outage is still acceptable. In this scenario, a momentary bump can be expected. During this bump, the valves, motors, and other devices might shutdown temporarily, and the sensors may not report back to the PLC systems during the bumps.

- Hot redundancy: is used when the process must not shut down for even a brief moment under any circumstances. In case of 'warm redundancy' or 'hot redundancy', two dedicated processors are connected in primary and standby configuration.

After initial and intermediate level simulation studies, model is designed with hot-redundancy features.

\section{Result and Discussions}

This section is divided into two sub-sections, namely, system reliability performance and experimental analysis of system failure. The algorithms are developed on MATLAB platform, is dedicated for testing system reliability and analyzing fault tree parameter, $\mathrm{T}_{\mathrm{Rmin}}$ of the proposed method. Process instrumentation of the proposed method is designed and developed using FT-ViewSE, a realtime industrial automation platform/simulator [2], [3].

\section{System Reliability Analysis}

Figs. 2(a) and 2(b) depict the two different sets of tests performed to evaluate system reliability performance of the proposed method vis-à-vis the other methods. The abscissa represents the samples collected at different within range threshold values of the component reliability. For example, the $10^{\text {th }}$ sample is at the highest limit of threshold value of component reliability of various components. The ordinate represents the system reliability performance of the proposed method (R5) over the methods (R2, R3, and R4). Figs. 2(a) and 1(b) are as well read in Table I. In Table I, the Col. A tabulates test samples 1 through 10 of Test I and Test II. Cols. $\mathrm{B}$ and $\mathrm{C}$ tabulate the system performance as a function of $F_{s}(t)$, the system failure of R5 versus R2, R3, and R4. Similarly, the Cols. D and E show the system performance of R5 as a function of $R_{s}(t)$, the system reliability as against system performance of $\mathrm{R} 2, \mathrm{R} 3$, and R4. The most important observation is at the $10^{\text {th }}$ test sample, where $R_{s}(t)$ is unique at the upper limits of the threshold values of various components. This unique observation can be seen in bold figures in the table. The relative difference between the reliability of R5 versus that of existing systems (R2, R3 and R4) shows improvement in R5 in terms of overall system reliability. This is established clearly that the system reliability of R5 is always better than that of R2, R3, and R4 as seen in histogram form in Fig. 2(a) and 2(b).

At upper limits of threshold values, the R5 has shown $\quad 32.8 \% \quad(=(0.9045-0.5760) \times 100)$ improvement in system reliability as against R2. Similarly, R5 has obtained $7.51 \%$ and $25.6 \%$ improvement as against that of R3 and R4 respectively. However, maximum improvement in system reliability of R5 when compared with that of R2 has varied from $35.1 \%$ to $40.5 \%$ as depicted in Table I and also in Fig. 2. Similarly, these values corresponding to improvement in reliability of R5 with respect to R3 and R4 have varied from $6.69 \%$ to $8.49 \%$ and $23.4 \%$ to $25.1 \%$ respectively. These values are highlighted in Table I. Thus, it can be summarized that the proposed $S^{2}$ HRS-HCS model exhibits superiority over all other existing models in terms of system reliability. 


\section{Evaluation of Chances of System Failure}

Here, $T_{R \min }$ which depends on $\beta$ is considered for evaluating system availability. It is observed that $\mathrm{T}_{\mathrm{Rmin}}$ of the R5 increase, and $\mathrm{h}(\mathrm{t})$ decreases with increase in $\beta$. Fig. 3 shows that the $T_{R \min }$ of R5 has attained the lowest values for all the $\beta$ 's between 1 to 2.4. $\mathrm{T}_{\mathrm{Rmin}}$ is examined extensively and found that, for system R5, the time required for repair is minimum compare to the time required for repair by the other systems. However, $\mathrm{T}_{\mathrm{Rmin}}$ by itself varies with different values of $\beta$ as shown in Fig. 3. For example, at $\beta=1$, the values of $\mathrm{T}_{\mathrm{Rmin}}$ are 31 days, 172 days, 71 days and 135 days for systems R5, R2, R3 and R4 respectively. For this, value of $\beta$ as 1 , $\mathrm{T}_{\mathrm{Rmin}}$ required for R5 is also minimum that is of 31 days in comparison to $\mathrm{T}_{\mathrm{Rmin}}$ of $\mathrm{R} 2, \mathrm{R} 3$ and $\mathrm{R} 4$. It is observed that the R5 has much lesser chances of failures as against chances of failure of the existing systems due to more if-else conditions.

\section{Conclusion}

Process C\&I model of the proposed HPU is designed with enhanced system reliability. The model can be extended its applications in automobile fuel stations by making appropriate changes in the design. At upper limits of threshold values, the proposed model (R5) has shown $(32.8 \%, 7.51 \%$, and $25.6 \%$ ) significant enhancement in system reliability as against that of the existing systems R2, R3 and R4 respectively. It has been shown that the proposed model has much lesser chances of failures than that of the existing systems due to more if-else conditions. The time required for repair $\left(T_{R \min }\right)$ is examined extensively and found that, for system R5, $\mathrm{T}_{\mathrm{Rmin}}$ is the minimum in comparison with the one of the existing systems R2, R3, and R4. Hence, the proposed model exhibits superiority over all other existing models in terms of system reliability. In this way, the R5 enhances the gainful application of the integrated gasification combined cycle power plants, and will have its direct impact on plant performance.

\section{References}

[1] J.J. Arnold and J.R. Capener, Modern Power Station Practice, Pergamon Press, $3^{\text {rd }} e$ e. incorporating Modern Power System Practice, Turbine, Generators and Associated Plant, British Electricity Int., London, Elsevier, Vol. 3, Chap. 6, pp. 491-495, 2008.

[2] G.R. Biswal, M.L. Dewal, and R.P. Maheshwari, "Process Control and Instrumentation of SeSWRS based Hydrogen Cooling System of A Thermal Power Station," IEEE Int. Conf. on Energy, Automation and Signal, Bhubaneswar, India, pp. 01-06, Dec. 28 - 30, 2011.

[3] G.R. Biswal, R.P. Maheshwari, and M.L. Dewal, "Modeling, Control and Monitoring of $\mathrm{S}^{3} \mathrm{RS}$ based
Hydrogen Cooling System in Thermal Power Plant," IEEE Trans. Industrial Electronics, Vol. 59, No. 1, pp. 562-570, Jan. 2012.

[4] K. Kawai, Y. Takizawa, and S. Watanabe, "Advanced Automation for Power-Generation Plants Past, Present and Future," Elsevier J. Control Engineering Practices, Vol. 7, pp. 1405-1411, Jun. 21, 1999.

[5] T. Samad, P. McLaughlin, and J. Lu, "System Architecture for Process Automation: Review and Trends," Elsevier J. Process Control, Vol. 17, pp. 191-201, Oct. 5, 2006.

[6] E. Zio, "Reliability Engineering: Old Problems and New Challenges," Elsevier J. Reliability Engineering and System Safety, Vol. 94, pp. 125-141, 2009.

[7] A. Muller, A.C. Marquez, and B. Iung, "On the Concept of E-Maintenance: Review and Current Research," Elsevier J. Reliability Engineering and System Safety, Vol. 93, pp. 1165-1187, 2008.

[8] R.L. Bannister, R.A. Newby, and W.C. Yang, "Hydrogen Fueled Power Plant," United States Patent 5,775,091, Jul. 7, 1998.

[9] K. Isokawa and K. Hisajima, "Electric Power Generator/Hydrogen Production Combination Plant," United States Patent 2007/0000251A1, Jan. 42007.

[10] A. Al-Ahmed, S. Hossain, B. Mukhtar, H. Abualhamayel, and J. Zaidi, "Hydrogen Highway: An Overview," Proc. IEEE Int. Energy Conf. and Exhibition, Manama, Bahrain, pp. 642-647, Dec. 18-22, 2010.

[11] K. Günter, H. Peter, and H. Franz, "Automotive Production of Liquid Hydrogen Storage Systems," Liquid Hydrogen Storage, Series Production, pp. 01-05, 2003.

[12] D.N.P. Murthy, M. Xie, and R. Jiang, "Weibull Models," John Wiley \& Sons, Series in Probability and Statistics, Part - G: Applications in Reliability, NY, USA, Nov. 2003.

[13] G.R. Biswal, R.P. Maheshwari, and M.L. Dewal, "Fault Tree Analysis and Process Instrumentation of $\mathrm{S}^{2} \mathrm{WRS}$ based Hydrogen Cooling System," Proc. IEEE Int. Energy Conf. and Exhibition, Manama, Bahrain, pp. 379384, Dec. 18-22, 2010.

[14] H.G. Hargrove, W. Montgomery, and J.R. Pipkin, "Control of Hydrogen Cooler Employed in Power Generators," United States Patent 5,097,669, Mar. 24, 1992.

[15] R. Blatter, P. Elkuch, K. Fischer, and E. Liebigand, "Generator Cooling System," United States Patent 6,112,544, Sep. 5, 2000.

[16] R.F. Brosnihan, T.J. Chenaille, J.T. Clark, S.D. Kilmartin, S.E. Kodesch, and R.A. Willams, "Skids, Modules, and Modular System for Monitoring HydrogenCooled Generators," United States Patent 7,448,252 B2, Nov 11, 2008.

[17] A. Almansoori and N. Shah, "Design and Operation of A Future Hydrogen Supply Chain Snapshot 
Model," Trans. IChemE, Part A, Chemical Engineering Research and Design, vol. 84, no. A6, pp. 423-438, Jun. 2006.

[18] I. Schulte, D. Hart, and R. Vorst, "Issues Affecting the Acceptance of Hydrogen Fuel," Int. J. Hydrogen Energy, Elsevier, vol. 29, pp. 677-685, 2004.

[19] W. Xiao, Y. Cheng, W.J. Lee, V. Chen, and S Charoensri, "Hydrogen Filling Station Design for Fuel Cell Vehicles," IEEE Trans. Industry Applications, vol. 47, no. 1, pp. 245-251, Jan./Feb. 2011 